\title{
L'hypospadias : aspects anatomo-cliniques et thérapeutiques au CHU de Conakry Guinée
}

\author{
Abdoulaye Bobo DIALLO', Ibrahima BAH'1, Balla Moussa TOURE2, \\ Lambert Bakary OUENDENO1, Ibrahima BALDE³, Mamadou Bobo DIALLO1
}

1 Service d'Urologie-Andrologie, Hôpital Ignace Deen, CHU de Conakry,

2 Service de Chirurgie pédiatrique, Hôpital Donka, CHU de Conakry,

3 Centre Mère et Enfants de Kipé, Conakry, Guinée.

RESUME

Objectif : Ressortir les aspects anatomo-cliniques et évaluer les résultats thérapeutiques des hypospadias suivis au CHU de Conakry.

Patients et Méthode : Il s'agit d'une étude rétrospective de type descriptif portant sur 60 cas d'hypospadias colligés durant une période de 6 ans allant du $1^{\mathrm{er}}$ janvier 2001 au 31 décembre 2006. Il s'agissait d'un échantillonnage exhaustif des cas colligés dans les services d'UrologieAndrologie et de Chirurgie pédiatrique du CHU de Conakry.

Résultats : L'âge moyen des patients était de 8 ans avec des extrêmes d'un jour et 33 ans. Quarante pour cent $(n=24)$ des patients avaient moins de 4 ans. Les patients de plus de 20 ans représentaient $16,7 \%(n=10)$ de notre échantillon. L'hypospadias était antérieur dans la moitié des cas, moyen dans 12 cas et postérieur dans 18 cas. La cryptorchidie, la hernie inguinale et la hernie ombilicale ont été les principales pathologies associées à l'hypospadias.

Sur le plan thérapeutique, $70 \%(n=42)$ des patients ont bénéficié d'une réparation en un temps et $30 \%(n=18)$ des cas d'une réparation en deux temps. Les techniques chirurgicales les plus utilisées étaient celles de Duplay (35\%), de Mathieu (25\%) et de Duckett $(16,7 \%)$. Les résultats thérapeutiques jugés après un recul moyen de 8 mois étaient bons dans $61,7 \%$ des cas, moyens dans $15 \%$ des cas et mauvais dans $23,3 \%$ des cas. La fistule urétro-cutanée (7 cas), la nécrose du lambeau ( 5 cas) constituaient les principales complications post opératoires rencontrées. Les meilleurs résultats ont été obtenus par la technique de Mathieu avec 9 bons résultats sur 15 .

Conclusion : L'hypospadias n'est pas exceptionnelle dans notre pratique quotidienne, cependant son traitement est émaillé de complications vues les conditions dans lesquelles ce type de chirurgie est exécutée dans notre pays. L'amélioration de nos résultats passe non seulement par la formation d'urologues pédiatres mais aussi par l'équipement de nos plateaux techniques en matériel de chirurgie microscopique.

Mots clés: hypospadias, urètre, urétroplastie

\section{INTRODUCTION}

L'hypospadias peut se définir comme une hypoplasie des tissus formant la face ventrale du pénis c'est à dire une immaturité des tissus situés à la face inférieure de la verge en aval de la division du corps spongieux [1].

La fréquence serait d'environ 1 hypospadias sur 300 naissances masculines et 1 sur 80 à 1 sur 100 dans les familles où existe déjà un enfant porteur de l'anomalie [14]. L'étiologie de cette malformation reste encore inconnue même si un certain nombre de travaux impliquent des désordres hormonaux, génétiques ou vasculaires $[1,7]$.

Le diagnostic essentiellement clinique de l'hypospadias semble aisé et le traitement demeure chirurgical avec son cortège de complications (des coudures persistantes, des fistules urétro-cutanées, des nécroses du lambeau, des sténoses au niveau de l'anastomose...). Les circoncisions

\section{Correspondance :}

Dr Abdoulaye Bobo DIALLO - Service d'Urologie-Andrologie, CHU de Conakry, BP 615, Conakry, République de Guinée Tel (224)60591615/(224)64372526 -

Email diallo_abobo@yahoo.fr 
rituelles pratiquées dans nos régions peuvent d'avantage compliquer cette chirurgie en raison de la perte du tablier préputial et de l'existence de cicatrices fibreuses.

Le but de ce travail était d'apprécier les aspects anatomocliniques et d'évaluer les résultats thérapeutiques des hypospadias suivis au CHU de Conakry.

\section{MATERIEL ET METHODES}

II s'agit d'une étude rétrospective de type descriptif portant sur 60 cas d'hypospadias colligés sur une période de 6 ans allant du $1^{\mathrm{er}}$ janvier 2001 au 31 décembre 2006. II s'agissait d'un échantillonnage exhaustif des cas colligés dans les services d'Urologie-Andrologie et de Chirurgie pédiatrique du CHU de Conakry.

Les critères d'inclusion étaient les patients hospitalisés et traités dans les services d'Urologie-Andrologie et de Chirurgie pédiatrique ayant un dossier médical comportant outre une observation médicale, un compte rendu opératoire et un suivi post opératoire d'au moins 3 mois. Ont été exclus de cette étude tous les cas d'hypospadias reçus avant ou après la période d'étude et ceux dont le dossier médical était inexploitable ou incomplet.

Dans le cadre du bilan, tous les patients ont bénéficié d'une échographie abdomino-pelvienne à la recherche d'anomalies associées à l'ectopie du méat urétral. Dans les cas sévères (hypospadias péno-scrotal, scrotal et périnéal) l'étude du caryotype n'a été réalisée que chez trois patients.

Les patients ont été opérés par différents chirurgiens sous anesthésie générale et les paramètres étudiés étaient d'ordre clinique et thérapeutique. Les résultats thérapeutiques ont été jugés selon la qualité du redressement de la verge, l'aspect cosmétique de la verge, la position du néo méat urétral, la perméabilité de l'urètre et l'existence ou non de fistule. Ainsi ont été qualifiés :

bons résultats : verge droite sans cicatrice disgracieuse avec un bel aspect cosmétique, un canal urétral perméable avec un méat urétral apical sans trouble mictionnel ;

résultats moyens : amélioration des anomalies initiales tant sur les plans urinaire que cosmétique, persistance d'une coudure, méat non apical, existence d'une fistule curable ultérieurement, nécessité d'une méatoplastie ou d'un cathétérisme dilatateur complémentaire ;

résultats mauvais : persistance de toutes les anomalies initiales, lâchage ou désunion des sutures et nécroses de lambeaux.

\section{RESULTATS}

En 6 ans, 60 cas d'hypospadias ont été colligés au CHU de Conakry dont $53,3 \%(n=32)$ au service d'Urologie-Andrologie et $46,7 \%(n=28)$ au service de Chirurgie pédiatrique. Les patients ont été amenés dans les services par leurs parents dans $55 \%(n=33)$ des cas, et ils nous ont été adressés par des médecins dans $45 \%(n=27)$ des cas. Seuls trois patients appartenaient à des familles où un cas d'hypospadias avait déjà été noté dans la fratrie.
L'âge moyen des patients était de 8 ans avec des extrêmes d'un jour et 33 ans. Quarante pour cent $(n=24)$ des patients avaient moins de 4 ans. Les patients de plus de 20 ans représentaient $16,7 \%(n=10)$ de notre échantillon. Neuf patients avaient déjà bénéficié d'une cure d'hypospadias et 7 autres de deux cures sans succès.

L'hypospadias était antérieur (Figure 1) dans la moitié des cas, moyen dans 12 cas et postérieur dans 18 cas (Tableau 1). La coudure prononcée de la verge, présente chez 26 patients, était la principale anomalie associée à l'ectopie du méat urétral. Une sténose du méat urétral ainsi qu'une torsion de la verge ont été notées dans 3 cas de part et d'autre. La cryptorchidie, la hernie inguinale et la hernie ombilicale ont été les principales pathologies associées à l'hypospadias (Tableau 2). Chez aucun patient il n'a été objectivé plus de deux pathologies associées à l'hypospadias.

Sur le plan thérapeutique, $70 \%(n=42)$ des patients ont bénéficié d'une réparation en un temps et $30 \%(n=18)$ des cas d'une réparation en deux temps, le premier temps ayant été consacré à la correction d'une coudure très marquée de la verge. Les techniques chirurgicales les plus utilisées (Figures 2 à 6) étaient celles de Duplay (35\%), de Mathieu (25\%) et de Duckett $(16,7 \%)$ (Tableau 3 ). La majorité des patients ( 57 cas) a bénéficié d'un drainage des urines vésicales soit par un cystocathéter $(51,7 \%)$ soit par une sonde urétrale $(43,3 \%)$. La durée moyenne de drainage était de 13 jours avec des extrêmes de 4 et 20 jours.

Les résultats thérapeutiques ont été jugés après un recul moyen de 8 mois (extrêmes de 3 et 22 mois) ; ainsi, ils étaient bons dans $61,7 \%$ des cas, moyens dans $15 \%$ des cas et mauvais dans $23,3 \%$ des cas. La fistule urétro-cutanée ( 7 cas), la nécrose du lambeau (5 cas) et la désunion des sutures (4 cas) ont été les principales complications post opératoires rencontrées (Tableau 4).

D'après la technique chirurgicale effectuée, les meilleurs résultats ont été obtenus avec la technique de Mathieu avec 9 bons résultats sur 15 . II a été également noté que les complications étaient plus nombreuses chez les patients ayant un drainage urinaire par sonde urétrale que ceux ayant bénéficié d'un drainage urinaire par un cystocathéter.

\section{DISCUSSION}

L'hypospadias est une malformation congénitale ayant des répercussions indéniables sur l'enfant et ses parents d'où la nécessité d'un diagnostic précoce et d'un traitement adéquat. Malheureusement, cette affection n'est pas bien connue dans nos contrées ce qui explique le retard du diagnostic.

En 6 ans nous avons recensé 60 cas d'hypospadias au $\mathrm{CHU}$ de Conakry, ce chiffre ne représente pas la totalité des hypospadias devant être pris en charge dans notre pays d'autant plus qu'il ne s'agit que de données purement hospitalières.

Nos patients ont consulté tard, l'âge moyen était de 8 ans et près de la moitié de notre échantillon avait atteint voire dépassé l'âge scolaire au moment de leur prise en charge.

Dans notre série, l'hypospadias était antérieur dans la moitiè 
Tableau 1 : Types d'hypospadias.

\begin{tabular}{lcc}
\hline Type de l'hypospadias & $\begin{array}{c}\text { Nombre } \\
\text { de patients }\end{array}$ & Pourcentage \\
\hline Glandulaire & 9 & 15,0 \\
Balano-pénien & 10 & 16,7 \\
Pénien antérieur & 11 & 18,3 \\
Pénien moyen & 12 & 20,0 \\
Pénien postérieur & 6 & 10,0 \\
Péno-scrotal & 5 & 8,3 \\
Scrotal & 4 & 6,7 \\
Périnéal & 3 & 5,0 \\
\hline Total & 60 & 100
\end{tabular}

Tableau 3 : Techniques chirurgicales utilisées.

\begin{tabular}{lcc}
\hline Techniques chirurgicales & $\begin{array}{c}\text { Nombre } \\
\text { de patients }\end{array}$ & Pourcentage \\
\hline Duplay & 21 & 35,0 \\
Mathieu & 15 & 25,0 \\
Duckett & 10 & 16,7 \\
Mustarde & 9 & 15,0 \\
Magpi & 5 & $\mathbf{8 , 3}$ \\
\hline Total & $\mathbf{6 0}$ & $\mathbf{1 0 0}$
\end{tabular}

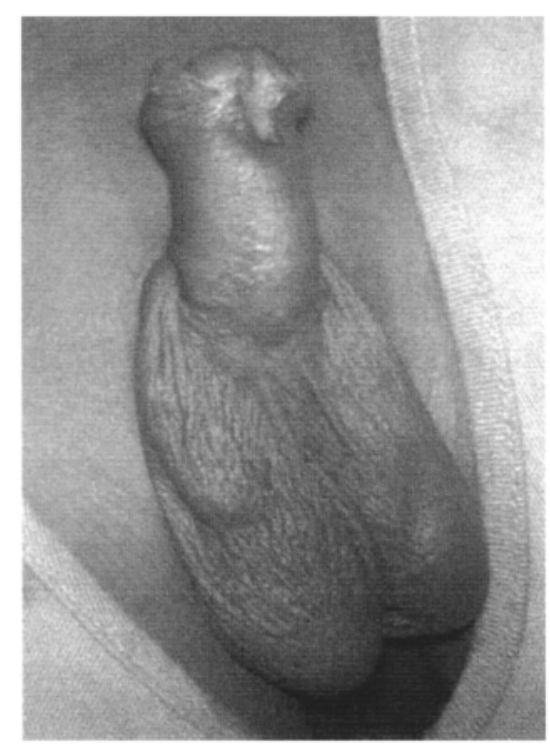

Figure 1: Hypospadias pénien antérieur
Tableau 2 : Pathologies associées à l'hypospadias.
Pathologies associées

Cryptorchidie gauche

Hernie inguinale

Hernie ombilicale

Omphalocèle

Micro pénis

Cryptorchidie droite + hernie ombilicale

Cryptorchidie gauche + hernie inguinale droite 1

Kyste du cordon spermatique
Nombre de patients

5

5

3

3

2

1

1
Tableau 4 : Fréquence des complications rencontrées.

\begin{tabular}{lcc}
\hline Complications & Nombre de cas & Pourcentage \\
\hline Fistule urétro-cutanée & 6 & 26,1 \\
Nécrose du lambeau & 5 & 21,7 \\
Désunion de la suture & 4 & 17,4 \\
Coudure résiduelle & 3 & 13,1 \\
Sténose au niveau de & & \\
l'anastomose & 2 & 8,7 \\
Sténose du méat & 2 & 8,7 \\
Hématome scrotal & 1 & 4,3 \\
\hline Total & 23 & 100
\end{tabular}

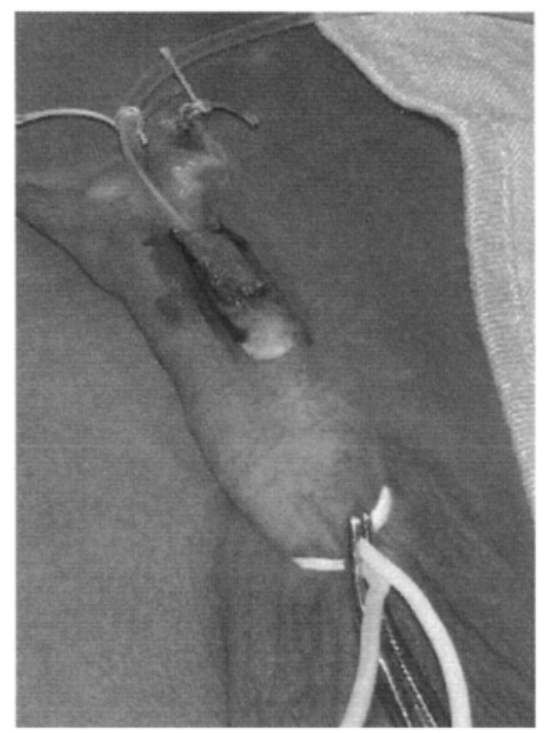

Figure 2 : Incision en "U" selon la technique de Mathieu. 


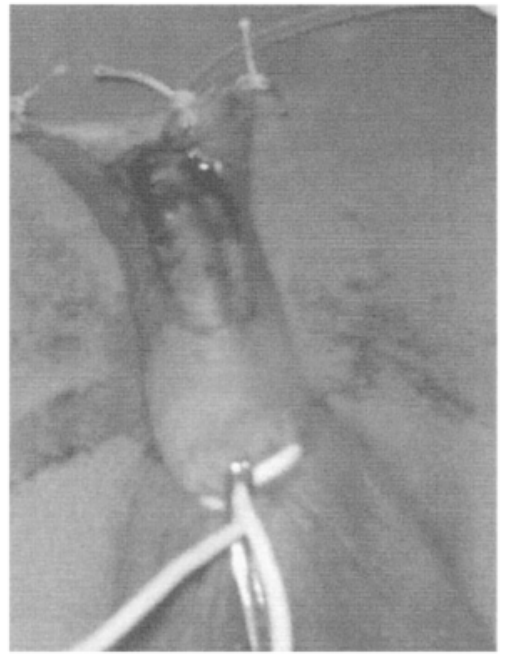

Figure 3 : Sutures latérales dans le but d'obtenir un néourètre par un lambeau de retournement.

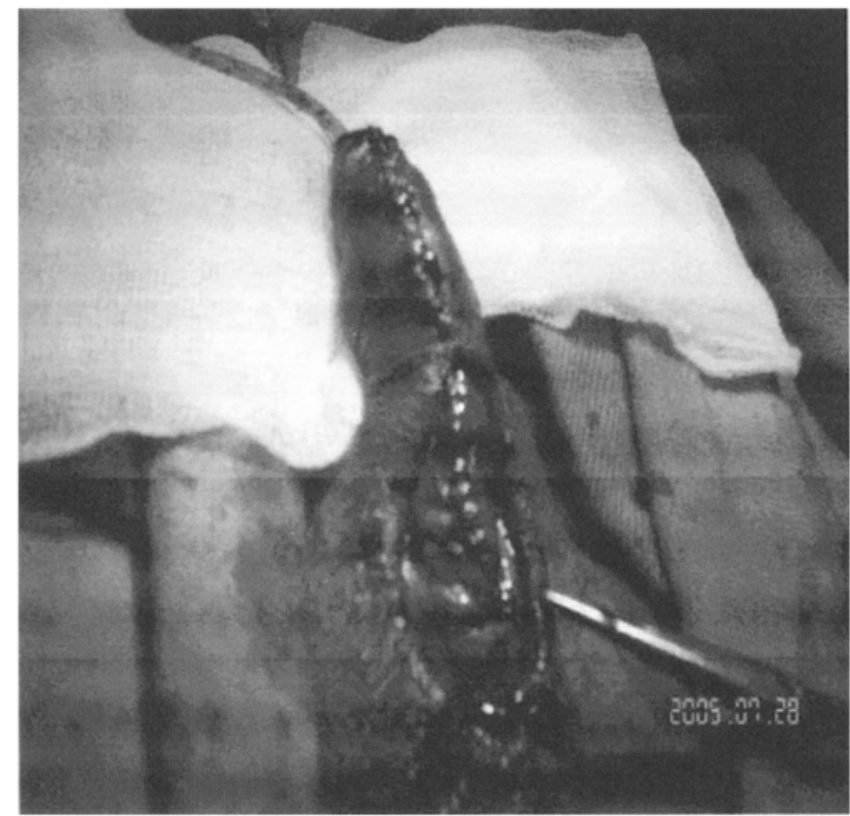

Figure 5 : Fermeture de la peau après urétroplastie selon la technique de Duplay.

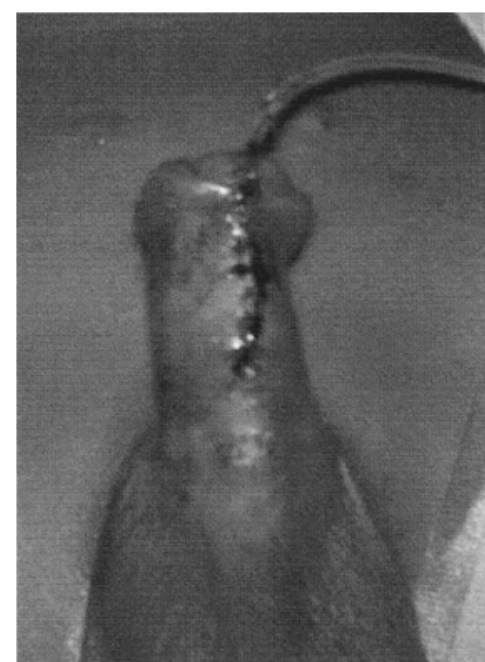

Figure 4 : Aspect final.

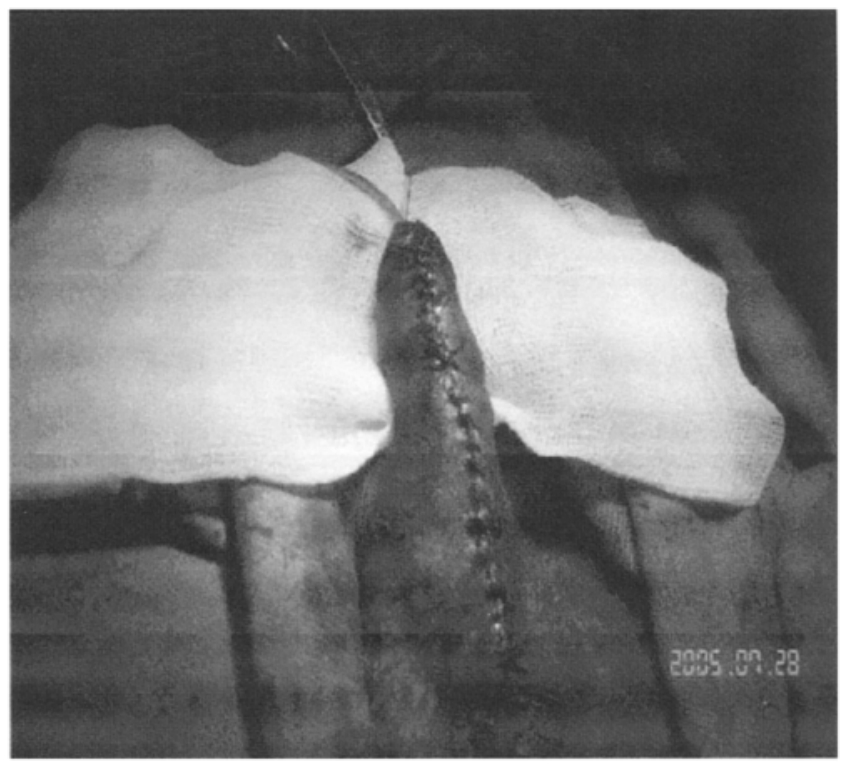

Figure 6 : Aspect final d'une urétroplastie selon la technique de Duplay. 
des cas ; cette forme antérieure représenterait 65 à $71 \%$ de l'ensemble des hypospadias [2,15]. La forme postérieure rencontrée chez 18 patients de notre série peut porter à confusion avec un état intersexué ; cependant quoique certains auteurs considèrent l'hypospadias comme une forme d'intersexe, ce n'est que dans les formes graves associées à une cryptorchidie bilatérale que la différentiation sexuelle doit être recherchée [6].

Dans ces cas, la détermination du caryotype, de l'AMH (Hormone anti-müllérienne) et le test à l'HCG deviennent indispensables, de même un génitogramme et une cystoscopie pourraient être utiles pour rechercher des restes müllériens [4]. II faut également préciser que la dysgénésie gonadique mixte (46 XY/45X mosaïque) et l'hermaphrodisme vrai $(80 \%$ $X X$ et $20 \%$ XY ou mosaïque) peuvent se rencontrer chez un hypospadias avec cryptorchidie uni ou bilatérale.

Dans notre étude, les pathologies associées à l'hypospadias étaient la cryptorchidie et la hernie inguinale, cela semble confirmer les données de la littérature [4].

Du point de vue thérapeutique, on dénombre de nos jours plus de 300 techniques opératoires des hypospadias. Elles sont toutes ingénieuses mais aucune d'elles n'est applicable à tous les types d'hypospadias. Actuellement la plupart des équipes chirurgicales s'accorde sur le traitement en un temps des hypospadias ; cependant $30 \%$ de nos patients ont été traités en deux temps. Ce traitement en deux temps n'a concerné que des patients ayant un hypospadias postérieur avec une coudure de verge prononcée ; cette première étape du traitement a consisté tout juste à un redressement de la verge et une correction d'éventuelles lésions associées telles une palmure de la verge.

Dans notre série, la technique de Duplay a été la plus utilisée et depuis quelques années cette technique a vu ses indications s'élargir, en particulier grâce à l'incision verticale de la gouttière urétrale décrite par Snodgrass [16]. Cet artifice a été effectué dans notre série chez 4 patients. Toutefois les techniques d'urétroplasties effectuées dans notre série ont toutes été pourvoyeuses de complications dont la plus fréquente était la fistule urétro-cutanée.

Nous pensons que la plupart des complications observées dans notre série serait imputable d'une part à une hémostase défectueuse, responsable d'hématome post-opératoire qui entraînerait la mise en tension des sutures prédisposant à l'apparition de fistules urétro-cutanées, et d'autre part à l'absence dans notre arsenal thérapeutique de lunettes grossissantes ou de microscope opératoire qui sont admis aujourd'hui par tous comme garants de meilleurs résultats $[12,13]$. En plus de cela, quelle que soit la technique d'urétroplastie utilisée, on doit tenter autant que possible de faire un plan intermédiaire sous cutané qui renforcerait l'étanchéité de la suture urétrale $[8,5]$. La réalisation de ce plan intermédiaire a fait l'objet de plusieurs publications, certains auteurs ont préconisé l'utilisation d'un lambeau cutané scrotal désépidermisé enfoui entre les plans urétral et cutané [10], d'autres ont opté pour un lambeau de vaginale [9] ou de dartos [3].
En ce qui concerne les modalités de drainage urinaire, au vu des résultats thérapeutiques obtenus dans notre étude, il nous parait plus nécessaire d'opter pour une dérivation sus pubienne que pour la classique mise en place d'une sonde urétrale qui pourrait faire le lit d'une éventuelle infection donc responsable d'un lâchage des sutures. Mitchel et al. [11] préconisent une dérivation urinaire suspubienne associée à un tuteur urétral en silastic intubant le néo urètre et l'anastomose sans atteindre la vessie.

Les résultats thérapeutiques ont été jugés bons dans $61,7 \%$ des cas, ce qui est inférieur aux résultats trouvés par Algallad et al. [2] et Samuel et al. [15] qui sont respectivement de $76 \%$ et $71 \%$.

\section{CONCLUSION}

L'hypospadias masculin ne constitue pas une pathologie exceptionnelle dans notre pratique quotidienne, cependant son traitement chirurgical demeure difficile et grevé de complications vues les conditions dans lesquelles ce type de chirurgie est exécutée dans notre pays.

L'amélioration des résultats obtenus dans cette étude passe par la formation d'urologues pédiatres aguerris à ce type de chirurgie d'une part, et par l'équipement de nos plateaux techniques en matériel de chirurgie microscopique.

\section{REFERENCES}

1. ALBERS N., ULRICHS C., GLUER S. et al. : Etiologic classification of severe hypospadias : implications for prognosis and managemant. J. Ped., 1997, $131:$ 386-392

2. ALGALLAD M.A. : Five years experience in hypospadias repair. Afr. J. Urol., 1999, 5 : 118-124.

3. CHURCHIILL B., VAN SAVAGE J., KHOURYA., Mc LORIE G. : The dartos flap as an adjunct in preventing uretrocutaneous fistlas in repeat hypospadias surgery. J. Urol., 1996, 156 : 2047-2049.

4. DE SY W.A., HOEBEKE P. : Considérations générales sur l'hypospadias. Ann. Urol., 1996, 30, 4 : 158-159.

5. DUBOIS R., PELIZZO G., NASSER H., VALMALLEA.F., DODAT H.: Les fistules de l'urètre après traitement chirurgical d'hypospadias. A propos d'une série de 74 cas. Prog. Urol., 1998, 8 : 1029-1034.

6. DUCKETT J.W. : Hypospadias. Campbell's urology 6 th ed. W. B. Saunders Co., 1963, 1893.

7. HUSMANN D.A. : Androgen receptor expression and penile growty during sexual maturation. Dial. Ped. Urol., 1996, $19: 1-8$.

8. KASS E., BOLONG D. : Single stage hypospadias reconstruction without fistula. J. Urol., 1990, 144, 520-522.

9. KIRKALI Z. : Tunica vaginalis : an aid in hypospadias surgery. Brit. J. Urol., 1990, $65: 530-532$.

10. LEE S., KIM K., KIM Y. : De-epithelialized scrotal flap in repair of urethrocutaneous fistula and hypospadias. Urology, $1990,36: 160-$ 163.

11. MITCHELL M.E., KULB T.B. : Hypospadias repair without bladder draining catheter. J. Urol., 1986, $135: 321$.

12. MOLLARD P., BASSET T., MURE P. : Traitement moderne de l'hypospadias. J. Urol., 1996, $102:$ 9-17

13. MONFORT G., DI BENEDETTO V. : Correction of hypospadias. Current Opinion in Urology, 1992, 2 : 414-419. 
14. PAPAREL P., MURE P.Y., MARGARIAN M., FEYAERTS A., MOURIQUAND P. : Approche actuelle de l'hypospadias chez l'enfant. Prog. Urol., 2001, 11 : 741-751.

15. SAMUEL M., CAPPS S., WORTHY A. : Distal hypospadias : which repair? Brit. J. Urol. Int., 2002, $90:$ 88-91.

16. SNODGRASS $W$. : Tubularized incised plate urethroplasty for distal hypospadias. J. Urol., 1994, 151 : 464-465.

Manuscrit reçu : février 2008 ; accepté avril 2008.

\section{ABSTRACT}

Anatomical, clinical and therapeutic features of hypospadias at Conakry teaching hospital, Guinea

Abdoulaye Bobo DIALLO, Ibrahima BAH, Balla Moussa TOURE, Lambert Bakary OUENDENO, Ibrahima BALDE, Mamadou Bobo DIALLO

Objective: To describe the clinicopathological features and evaluate the results of treatment of hypospadias at Conakry teaching hospital.

Patients and Method: This retrospective descriptive study was based on 60 cases of hypospadias observed over a 6year period from 1st January 2001 to 31 December 2006, corresponding to all cases seen in the Urology-Andrology and Paediatric Surgery departments of Conakry teaching hospital.

Results: The mean age of the patients was 8 years (range: one day to 33 years). Forty per cent $(n=24)$ of patients were less than 4 years old. Patients over the age of 20 represented $16.7 \%(n=10)$ of this sample. Hypospadias was anterior in one half of cases, middle in 12 cases and posterior in 18 cases. Cryptorchidism, inguinal hernia and umbilical hernia were the main diseases associated with hypospadias.

Seventy per cent $(n=42)$ of patients were treated by onestage repair and $30 \%(n=18)$ were treated by two-stage repair. The surgical techniques most frequently used were those of Duplay (35\%), Mathieu (25\%) and Duckett (16.7\%). The results of treatment, assessed after a mean follow-up of 8 months, were good in $61.7 \%$ of cases, moderate in $15 \%$ of cases and poor in $23.3 \%$ of cases. Urethrocutaneous fistula (7 cases) and flap necrosis (5 cases) were the main postoperative complications. The best results were obtained by the Mathieu technique with 9 good results out of 15 cases.

Conclusion: Hypospadias is not exceptional in our daily practice, but its treatment is associated with complications related to the conditions in which this type of surgery is performed in our country. Improvement of our results requires not only training of paediatrician urologists but also acquisition of microscopic surgery equipment.

Key words: hypospadias, urethra, urethroplasty 